\title{
Coordinated Control Object Modeling Based on Improved Particle Swarm Optimization Algorithm
}

\author{
Guanggang Shang ${ }^{1,}$ a, Lincang Ju ${ }^{2, b}$, Li Tang ${ }^{3, c^{*}}$ and Yongjie Gao ${ }^{4, d}$ \\ ${ }^{1}$ Shandong Electric Power Engineering Consulting Institute Corp, 106th Minziqian Road Jinan \\ 250013, Shandong, China \\ ${ }^{2,3,4}$ Xi'an Jiaotong University,28th Xianning West Rd, Xi'an 710049, China \\ a Shangguanggang@sdepci.com, ${ }^{\mathrm{b}}$ licang@mail.xjtu.edu.cn, ${ }^{\mathrm{a}}$ tangli341@stu.xjtu.edu.cn, \\ d myjdgaoyj@163.com
}

\begin{abstract}
Keywords: Supercritical units; Coordinated control object; Particle swarm optimization; Model
Abstract. In this paper, a modeling method of coordinated control object of supercritical power plant unit is studied. Combined with the actual operating history data of the power plant, by using experimental modeling method, this paper firstly gets the modeling structural according to the dynamic characteristics of the object, and then using the improved particle swarm optimization algorithm to get the unknown parameters of model, and ultimately determines the model of unit coordinated control object. The verification by operating data shows that the established model has high precision and control strategy can be further studied based on this model.
\end{abstract}

\section{Introduction}

Supercritical unit coordinated control object with multi-variable, strong coupling and nonlinear characteristics makes more difficult to establish accurate mathematical model. Researching how to build a supercritical unit coordinated control object model is of great significance. Researchers at home and abroad make a lot of research on modeling of the supercritical unit coordinated control object. According to the modeling principle, it mainly divides into two methods: mechanism modeling and experimental modeling[1]. Modeling experiment method is also known as test modeling method, namely supercritical unit based on the actual operation process of input and output data, simulation experiments by assuming model, matching with the input/output data of the model according to the experimental results. Experimental modeling method does not require deep understanding of the object, and modeling process is simple. Generally the proposed model in the form of a written transfer function has a wide range of uses, so experimental modeling method is adopted to establish the model of the unit.

\section{Modified Particle Swarm Optimization Algorithm}

Compared with other evolutionary algorithms such as genetic algorithm, Particle Swarm Optimization algorithm principle is simple, easy programming, running speed, and have less control parameters in program internal, reducing the running effects of the program because of improper parameter settings and having better ability of global optimization. Since the PSO algorithm was proposed, it has been widely applied in the optimization problem of various fields[2-4].However, after the particle initial position and speed determined, the change of them is limited in a small range, so if using PSO algorithm to solve practical problems, for the established outside of the area, the particle cannot be searched, thus the problem could not well solved which limited the application of the algorithm.

The study of swarm intelligence algorithm shows that whether a group has enough intelligence depends on whether the individuals in the group evolution have great randomness. If a group of individuals in the process of evolution has always been a certain amount of change required to limit in a small scope, then the group is difficult to achieve greater intelligence; If individuals in the group has strong randomness, the development of the group will have infinite possibility and it is easy to obtain 
the optimal evolution direction, then the group achieve strong intelligence. Because of quantum space particles with a strong randomness, the groups made of particles which with motion law of microcosmic space midpoint have strong intelligence. The combination of the movement of particles in quantum space and the PSO algorithm get the Quantum-behaved Particle Swarm Optimization algorithm [5].

\section{The Determination of Model Structure}

System identification consists of two parts--structure identification and parameter identification. By combining with the dynamic property and prior knowledge of the controlled object ,the controlled object model structure form is determined, and then on the basis of the model structure, the difference between the actual output values and the output value of the identify model is continually reduced .Finally get most conforms to the models and features of the object.

According to the basic principle of supercritical unit coordinated control system, generally supercritical unit coordinate control object can be simplified to a three inputs and three outputs system. Three inputs amount are respectively the steam turbine control valve opening ${ }^{\mu_{T}}$, fuel quantity $B$ and feed water flow $W$. Three outputs are respectively unit power $N$, the main steam pressure $P$ and intermediate point temperature $T$. The structure of the supercritical unit coordinated control object is shown in figure 1.

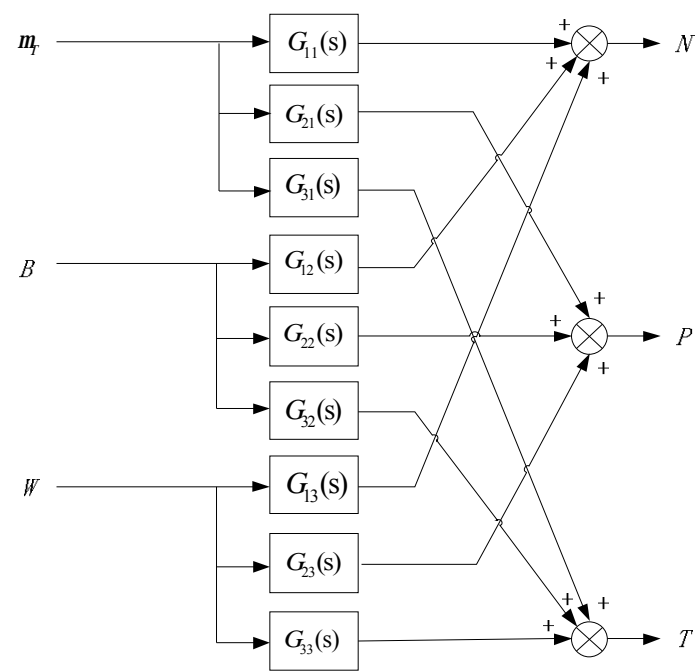

Fig.1 Simplified structure graph of coordinated control system

\section{Modeling Procedures}

System identification based on QPSO algorithm which is on the basic of input data and output data of the controlled system, according to the selected evaluation criterion, the model which is most close to the object features is selected from a set of mathematical models. Its basic principle is shown in figure 2.

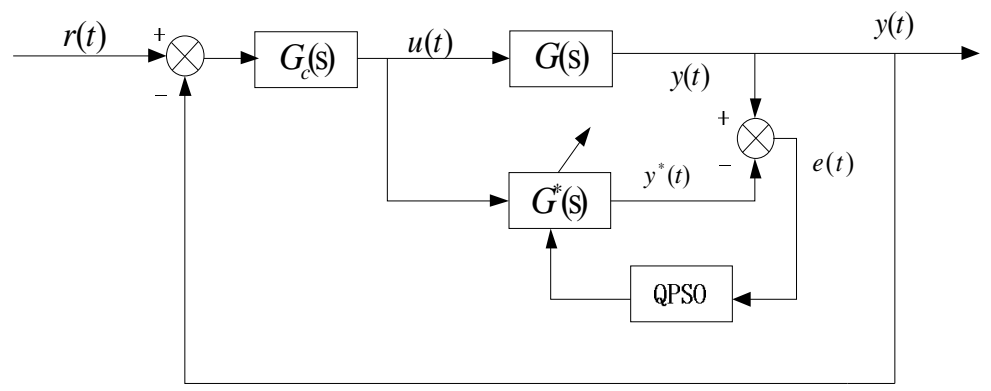

Fig.2 The principle diagram of the system identification using QPSO algorithm 
In figure2, $G(\mathrm{~s})$ is the known actual transfer function model, $G^{*}(\mathrm{~s})$ is the selected identification transfer function model, $G_{c}(\mathrm{~s})$ is controller, and here the PID controller is adopted. The input signal of actual transfer function model $G(\mathrm{~s})$ and identification the transfer function model $G^{*}(\mathrm{~s})$ is the same and both of them are the controller output signal $u(t)$.In figure, $e(t)=y(t)-y^{*}(t), e(t)$ is difference of output value between the actual model and the identification model. According to the size of $e(t)$, QPSO algorithm adjust the parameters of the identification model $G^{*}(\mathrm{~s})$, making the identification model $G^{*}(\mathrm{~s})$ gradually close to the actual model $G(\mathrm{~s})$.

In order to better evaluate the approaching degree of the identification model and the actual model, Mean Squared Error (MSE) is introduced as the evaluation criterion of system identification. MSE refers to the average of the sum of squares of a set of data parameters' difference between the estimated value and the actual value. The smaller the MSE, the better identification effect, which shows that system identification model is closer to the actual model. The calculation formula of MSE is shown as below:

$$
M S E=\sqrt{\frac{\varepsilon_{1}^{2}+\varepsilon_{2}^{2}+\mathrm{L}+\varepsilon_{n}^{2}}{n}}=\sqrt{\frac{\sum \varepsilon_{i}^{2}}{n}},
$$

where $\varepsilon_{i}$ is the difference between parameters estimated value and the actual value; $n$ is the number of parameters.

According to the power plant practical operation data, the process which uses quantum-behaved particle swarm optimization algorithm for the controlled object model to system identification is shown as follows:

1) First of all, we select the appropriate identification input and output data from the power plant database and then we make the data preprocessing using the actual production data smoothing processing method.

2) Through the analysis of the dynamic characteristics of controlled object to be identified, the structure form of identification model is determined.

3) For the selected object model structure, we determine the number of parameters needed to identify, set the initial value of parameters and parameter optimal range, and determine program control parameters such as quantum particle swarm optimization algorithm of particle population size and the maximum iterations number of the Identification program.

4) Comprehensively utilizing MATLAB and Simulink environment, combined with the power plant operation data, we can calculate the fitness value of current parameters algorithm.

5) Comparing the relationship between the current parameters fitness value and the best fitness value, then we update the position of the particle and get the newest parameter values. The fourth and fifth steps are cycled until it reaches the maximum number of iterations or meets the requirements of fitness value.

6) We need record the parameter optimal value and corresponding fitness value under this model order, adjust the model order and continue to run the program again from the second step.Then we can continue the same steps for all possible order model for parameters optimization.

7) Compared to performance index of different order model, we select the most optimal model, thus determining the structure and parameters of the model.

\section{Model Building Based on the Actual Data}

In this paper, used data are come from a $600 \mathrm{MW}$ unit whose boiler is supercritical parameter variable pressure spiral pipe once-through boiler of SG - 2084/25.4 - M979 type made in Shanghai boiler works Ltd. The boiler is a single furnace, a reheat and a solid-state discharge semi-outdoor Layout; Turbine has a supercritical pressure, a reheat, single shaft, three cylinder four type exhaust steam turbine of TC4F - 26-24.4/566/566 type made in Dong Fang Steam Turbine Works. Making use 
of 600 groups operation historical data selected from power plant DCS database whose load range is $400 \mathrm{MW}$ to $500 \mathrm{MW}$, we can proceed system identification for supercritical unit coordinated control object. Of the selected data, the units of feed water flow, coal feed, the steam turbine control valve opening, the unit power, main steam pressure, intermediate point temperature are respectively $\mathrm{t} \cdot \mathrm{h}^{-1}$, $\mathrm{t} \cdot \mathrm{h}^{-1}, \%, \mathrm{MW}, \mathrm{MPa},{ }^{\circ} \mathrm{C}$. The sampling period of each parameter is $6 \mathrm{~s}$. The former 300 groups data are used to identify the coordinated control object and the later 300 groups are used to verify the identification model.

First we will carry out smooth processing on the five point three times filtering theory introduced by the previous section using the selected 600 groups data, and then use MATLAB preparation QPSO identification, combine with the former 300 groups of data and the Simulink simulation platform building model for system identification. Among them, the population size in QPSO program is 20, the largest number of iterations is 500, the model identification of Simulink platform use the model structure in section 3.3.1 according to object dynamic behavior, the range of the amplification coefficient $\mathrm{K}$ in the transfer function model is set to [0,200], the scope of parameters optimization of time constant is set to $[0,600]$. The unknown parameters of transfer function through identifying are listed in table 2.

Table 1 the identification results of coordinated control object

\begin{tabular}{lllll}
\hline Parameter & $K$ & $T_{1}$ & $T_{2}$ & $T_{3}$ \\
\hline$G_{T W}(s)$ & 0.16 & 180.1 & 254.1 & - \\
$G_{T B}(s)$ & 2.28 & 10.2 & 165.9 & 576.7 \\
$G_{T \mu_{T}}(s)$ & 8.73 & 140.5 & 190.8 & 462.3 \\
$G_{P_{T} W}(s)$ & 2.5174 & 126.2 & 128.4 & 147.4 \\
$G_{P_{T} B}(s)$ & 0.1237 & 282 & 400.1 & - \\
$G_{P_{T} \mu_{T}}(s)$ & 0.7667 & 100.5 & 120 & - \\
$G_{N W}(s)$ & 62.8 & 81.3 & 103.8 & 122.9 \\
$G_{N B}(s)$ & 2.497 & 186.8 & 202.7 & 208 \\
$G_{N \mu_{T}}(s)$ & 150 & 35.4 & 36.7 & -
\end{tabular}

Parameter data in table 2 used the former 300 sets of data from selected 600 groups data to identify. In order to verify the accuracy of identification results, now we use the later 300 groups of data for validation. Corresponding to the three parameters' actual value which are the steam turbine control valve opening, coal feed, feed water flow, correlation curve between practical value of power unit and the output value shown in table 2 to identify the model are shown in figure 3 . The mean square error value between output power system got through the identification model and the actual power data is 2.8344, achieving good recognition effect.

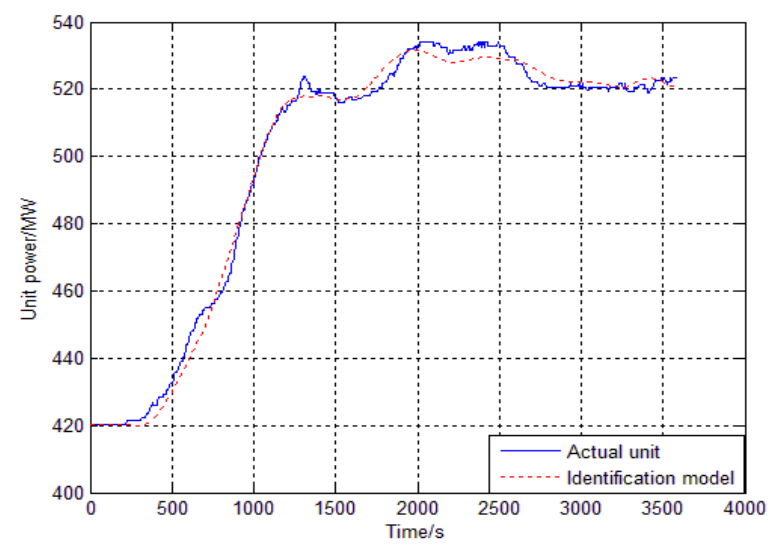

Fig.3 The contrast curve between unit power actual value and the identification model output value 
The correlation curve between practical value of main stream pressure and the output value shown in table 2 to identify the model are shown in figure 4. The mean square error value between the main stream pressure got through the identification model and the actual data is 0.1624 , with a higher accuracy of identification of transfer function.

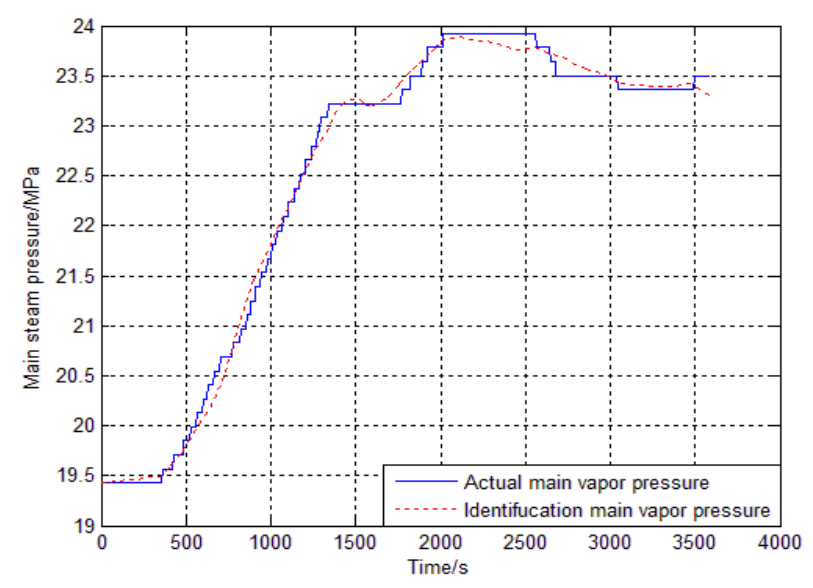

Fig.4 The contrast curve between main steam pressure actual value and the identification model output value

The correlation curve between practical value of Intermediate point temperature and the output value shown in table 2 to identify the model are shown in figure 5. The mean square error value between intermediate point temperature got through the identification model and the actual power data is 2.2326, achieving good recognition effect.

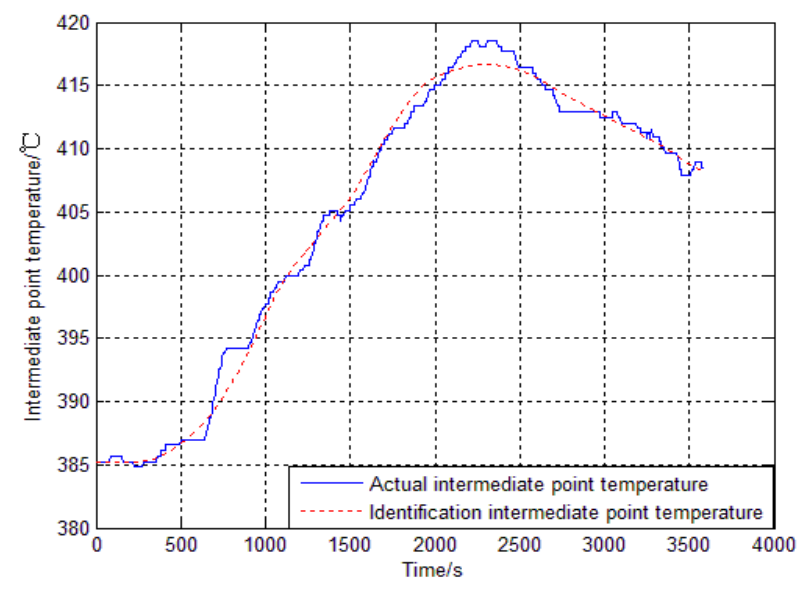

Fig.5 The contrast curve between intermediate point temperature actual value and the identification model output value

\section{Conclusion}

At first, aiming at the shortcomings of the existing particle swarm optimization algorithm, we combined the principle of quantum mechanics and the particle swarm optimization algorithm. Using MATLAB programming got the quantum-behaved particle swarm optimization algorithm, by improving the algorithm's practicality and the solving precision, and the effectiveness the common function on the algorithm is verified. And then combined with system identification of some actual data of 600MW supercritical unit, we got linear mathematical model of load interval from 400MW to $500 \mathrm{MW}$ in the unit coordinated control object. By the mean square error value and contrast curve between three parameters' actual value which are unit power, main steam pressure and intermediate point temperature unit power, main steam pressure and intermediate point temperature and the identifying model output value, we can see that for using quantum-behaved particle swarm optimization algorithm combined with the actual operation data obtained by system identification of 
coordinated control object model, corresponding to the actual input data, the actual output curve and identification the model output curve alignment is higher, the mean square error of actual output data and identification model output data is smaller, with a high accuracy identification model. We can go on the further research of the control strategy based on the identification model.

\section{References}

[1] Kesong Mi, Bo Wang, Jianmeng Yang, Modeling and Simulation of Thermal Object in Water of 600MW Supercritical Unit Control Based on System Identification. Application of Energy Technology, 2011, 11(9): 26-29.

[2] Hongyan Zhang. The Research of PID Controller Based on Improved PSO Algorithm [J]. China Test, 2013, 39(5): 96-98.

[3] Junxue Wu. The Research of BP Neural Network Based on PSO-EO Optimization Algorithm [J]. Science Technology and Engineering, 2010, 10(24): 6047-6049.

[4] Yanbing Tian. Research of Self-tuning PID Temperature Control Based on PSO [J]. Control and Instruments in Chemical Industry, 2010, 37(11): 31-33.

[5] Xiaojun Wu, Jun Sun. Quantum Behaved Particle Swarm Optimization: Principles and Applications [M]. Beijing: Tsinghua University Press, 2011.

[6] Lincang Ju, Qishou Liu, Qingyu Yang. The Power Plant Thermal Process Control System [M]. Xi'an: Xi'an Jiao Tong University Press, 2009. 